\title{
Efecto de la fertilización edáfica en el crecimiento y desarrollo de Phaseolus vulgaris cv. ICA Cerinza
}

\section{Effect of edaphic fertilization on the growth and development of Phaseolus vulgaris cv. ICA Cerinza}

DANIEL BAUTISTA-ZAMORA', 2

CARMEN CHAVARRO-RODRÍGUEZ'

JEIMMY CÁCERES-ZAMBRANO'

SEBASTIÁN BUITRAGO-MORA'

Frijol Cerinza en la granja Marengo

Foto: D. Bautista-Zamora

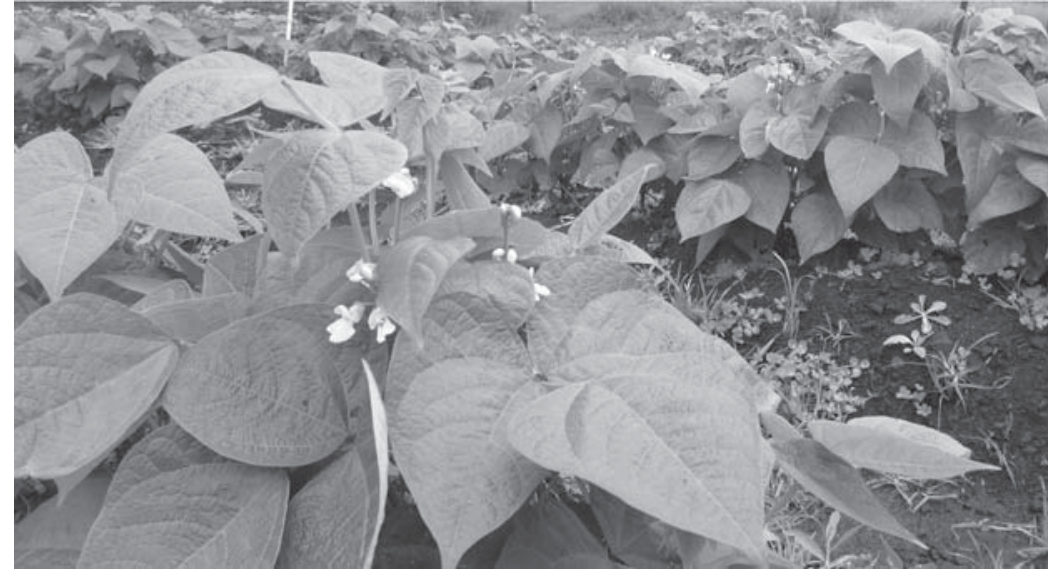

\section{RESUMEN}

La nutrición de cultivos es un eje central en la producción agrícola, sin embargo, en ocasiones las adiciones de fertilizantes no corresponden a las condiciones edáficas del área de cultivo, ni a los requerimientos por parte de la planta; este inadecuado manejo de la fertilidad degrada el suelo, disminuye el rendimiento y aumenta los costos de producción. Una alternativa para conservar los suelos, es la aplicación de enmiendas orgánicas, las cuales aumentan la disponibilidad de nutrientes y dan lugar a la recuperación de suelos. El objetivo del presente trabajo, fue determinar el efecto en el crecimiento y desarrollo de Phaseolus vulgaris var. Cerinza con la aplicación de enmiendas orgánicas (compost y lombricompost) y fertilizante comercial. Se evaluó la altura de la planta, el contenido de pigmentos fotosintéticos, número de hojas y estructuras reproductivas, materia seca, peso fresco, área foliar, e índices de crecimiento: área foliar específica (AFE), tasa de crecimiento de cultivo (TCC) y tasa de asimilación neta (TAN). Los resultados a los 56 días después de siembra mostraron diferencias significativas en altura, en donde los tratamientos de compost y lombricompost, presentaron mayores valores con relación a la fertilización comercial; en otras variables de crecimiento no hubo diferencias significativas entre tratamientos.

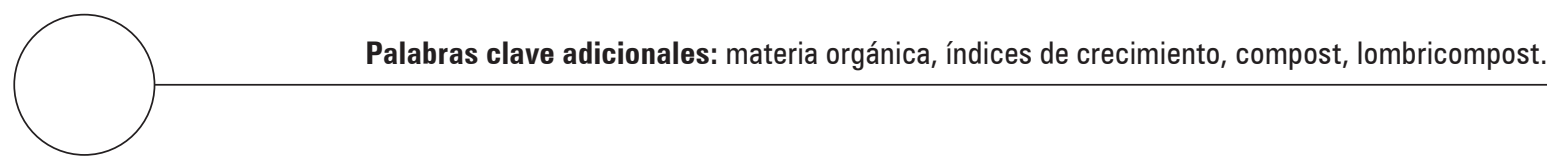

\footnotetext{
Facultad de Ciencias Agrarias, Universidad Nacional de Colombia, Bogotá (Colombia).

2 Autor para correspondencia. dambautistaza@unal.edu.co
} 


\section{ABSTRACT}

Crop nutrition is a central axis in agricultural production; however, sometimes fertilizer additions do not correspond to the edaphic conditions of the area of cultivation or the requirements of the plants; this inadequate fertilization management induces soil degradation, decreased yield and high production costs. An alternative to soil conservation is the application of organic amendments, which increase the availability of nutrients and give rise to the recovery of soils. The aim of this study was to determine the effect on the growth and development of Phaseolus vulgaris var. Cerinza with the application of organic amendments (compost and vermicompost) and commercial fertilizer. Plant height, photosynthetic pigment content, number of leaves and reproductive structures, fresh and dry weight, leaf area, area under the canopy and growth indexes: specific leaf area (SLA), crop growth rate (CGR) and net assimilation rate (NAR) were evaluated. The results at 56 days after sowing showed significant differences in height, where the treatments of compost and vermicompost had higher values in relation to the commercial fertilization. In the other growth variables, there were no significant differences between the treatments.

Additional key words: organic matter, growth indexes, compost, vermicompost.

Fecha de recepción: 17-10-2016 Aprobado para publicación: 15-04-2017

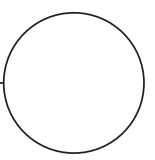

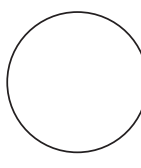

El frijol (Phaseolus vulgaris L.) es una especie leguminosa de origen mesoamericano (Miranda, 1967; Hernández et al., 2013), apreciada por propiedades nutricionales como el alto contenido proteico y aporte de carbohidratos, vitaminas y minerales en menor proporción (Ulloa et al., 2011), razón por la cual esta especie se ha categorizado como uno de los cultivos obligatorios en programas de seguridad alimentaria (Fenalce, 2004). Se estima que en Colombia aproximadamente 120.000 familias se dedican al cultivo de frijol, principalmente en departamentos como Antioquia, Santander, Huila, Tolima, Boyacá, Cundinamarca y Nariño (Fenalce, 2004).

Las variedades de frijol en Colombia son clasificadas de acuerdo a su hábito de crecimiento en volubles y arbustivas, de estas, las variedades volubles aportan el $65 \%$ al total de la producción nacional (Santana y Mahuku, 2002), no obstante, estas variedades tienen mayores costos de producción representados en mano de obra y materiales (43\%) debido a las labores de tutorado (Arias et al., 2007). Igualmente, labores de manejo agronómico como la fertilización, contribuyen a un aumento de costos, pues en el cultivo de frijol la inadecuada aplicación de fertilizantes se evidencia en la pérdida de nutrientes como el nitrógeno y el fósforo (Coral y Molina, 2010) que a su vez tiene consecuencias ambientales.

La aplicación inadecuada de abonos químicos a largo plazo ha traído daños ambientales para el suelo tales como la pérdida de fertilidad, reducción de la biodiversidad y contaminación, sumando al deterioro de las aguas subterráneas, lo cual afecta la sostenibilidad de los sistemas agrícolas y contribuye a la disminución de la productividad (Hernández et al., 2014). Por el contrario, los fertilizantes orgánicos edáficos pueden ser un valioso restaurador del suelo y fuente de nutrientes para las plantas, además de mejorar el rendimiento y las propiedades del suelo, como la capacidad de retención de agua, porosidad, disminución de la densidad aparente (Edwards y Lofty, 1982; Schjonning y Christensen, 1994; Bell, 1993; Kapkiyai et al., 1999; Díaz et al., 1999; Kwabiah et al ., 2003), estabilización del pH del suelo, aumento de la biomasa microbiana (Leifeld et al., 2002; Jedidi et al., 2004), mayores concentraciones de nutrientes disponibles para las plantas y reducción de enfermedades (Hoitink y Boehm, 1999); Sin embargo los aumentos de materia orgánica en el suelo ocurren lentamente (Drinkwater et al., 1995; Wander et al ., 1994).

Aunque las enmiendas orgánicas proporcionan la mayor parte de los elementos esenciales para la planta, la disponibilidad limitada y calidad variable, no suministran cantidades suficientes para satisfacer la demanda del cultivo (Gentile et al., 2010; Palma et al ., 1997 ), incluso el uso de recursos orgánicos de baja calidad inmoviliza nutrientes (Palma et al ., 1997 ), por otra parte, la fertilización mineral permite una rápida disponibilidad de elementos para la planta, favoreciendo algunas variables de crecimiento y rendimiento, lo 
cual depende de las condiciones ambientales, debido a que este tipo de fertilización tiene una rápida mineralización y adsorción al suelo, además de una frecuencia de aplicación mayor, debido a su alta solubilidad y precipitación (Citak y Sonmez, 2010). Sin embargo tanto en el frijol arbustivo como en el frijol voluble, la materia orgánica tiene un efecto significativo en el rendimiento del grano (Coral y Molina, 2010), por tanto las estrategias de manejo no pueden omitir esta variable.

Jácome et al. (2013) evaluaron el efecto de dos dosis de lombricompost en Phaseolus vulgaris aplicando $1 \mathrm{t} \mathrm{ha}^{-1}$, $5 \mathrm{t}$ ha $^{-1} \mathrm{y}$ una combinación de lombricompost y fertilizante de síntesis química (NPK 10-30-10), en dosis de $5 \mathrm{t} \mathrm{ha-1}$ y $300 \mathrm{~kg} \mathrm{ha}^{-1}$, respectivamente. Observaron que la aplicación de lombricompost regula el $\mathrm{pH}$, la estructura del suelo y la capacidad de intercambio catiónico, finalmente la aplicación de lombricompost combinado con el fertilizante inorgánico mostró los mejores resultados, tanto en las propiedades del suelo como en el rendimiento de grano, no obstante, los autores consideran que es necesario evaluar la aplicación de mayores dosis de lombricompost para tener mejores resultados con el fin de disminuir la fertilización de síntesis química y por tanto, los costos de producción. Investigaciones relacionadas muestran que con dosis de compost por encima de 4,8 $\mathrm{t} \mathrm{ha}^{-1}$ mejoran variables como número de vainas, número de hojas, área foliar y propiedades del suelo tales como el $\mathrm{pH}$ y la conductividad hidráulica (Alemán, 2006).

El objetivo de la presente investigación fue determinar el efecto de la aplicación de compost, lombricompost y una combinación comercial de N-P-K en el crecimiento y desarrollo de plantas de frijol (Phaseolus vulgaris var. Cerinza), a través del análisis de la fenología, el contenido de clorofila, distribución de materia seca, área foliar e índices de crecimiento.

\section{MATERIALES Y MÉTODOS}

\section{Material vegetal y establecimiento del experimento}

El experimento se llevó a cabo en el área de invernaderos de la Facultad de Ciencias Agrarias de la Universidad Nacional de Colombia, sede Bogotá, con altitud de $2.640 \mathrm{msnm}$, temperatura entre 14 y $25^{\circ} \mathrm{C}$ y humedad relativa de 68 a 72\%. Se emplearon semillas de Phaseolus vulgaris var. Cerinza proporcionadas por el obtentor, Universidad Nacional de Colombia. Se realizaron 16 camas de 4,0 × 1,20 m con $1 \mathrm{~m}$ de separación entre camas y una distancia entre plantas de 40 $\mathrm{cm}$, para un total de 18 plantas por cama. Se emplearon como fuentes fertilizantes Triple 15 (N 15\%, P $15 \%$ y K $15 \%$ ), lombricompost (N 1,17\%; P 1,16\%; K $0,37 \%$ y Ca $2,1 \%$ ) y compost ( $\mathrm{N} 1,1 \% ; \mathrm{P}_{2} \mathrm{O}_{5} 2 \% ; \mathrm{K}_{2} \mathrm{O}$ $2,4 \%$ y $\mathrm{CaO} 3 \%$ ), estos dos últimos proporcionados y caracterizados por la Facultad de Ciencias Agrarias, Universidad Nacional de Colombia, Bogotá. Estas fuentes fertilizantes corresponden a los tratamientos evaluados durante el estudio: T1: testigo (ningún tipo de fertilización), T2: compost, T3: lombricompost y T4: Triple 15. Las dosis empleadas por tratamiento fueron $9,2 \mathrm{t} \mathrm{ha}^{-1}$ de lombricompost, $10 \mathrm{t} \mathrm{ha} \mathrm{a}^{-1}$ de compost y $0,7 \mathrm{t} \mathrm{ha}^{-1}$ de Triple 15 .

La fertilización orgánica se realizó 12 d antes de la siembra, con el fin de que se incorporará al suelo; y la fertilización química se llevó a cabo en el momento de la aparición de las dos primeras hojas verdaderas a los 15-20 d después de siembra (dds). Se ejecutaron cuatro muestreos destructivos cada ocho días a partir de los 31 dds, tomando cuatro plantas al azar de cada tratamiento para así realizar las mediciones de las variables.

\section{Análisis de crecimiento y desarrollo}

Se determinó el número de hojas y la altura de la planta $(\mathrm{cm})$, midiendo desde la base del tallo hasta el meristemo apical de la planta. Se realizaron mediciones del peso fresco de hojas-tallos y botones florales, y el peso seco del material luego de su secado en una estufa a $65^{\circ} \mathrm{C}$ hasta peso constante. El área foliar se determinó empleando un medidor de área foliar LI-COR 3100 (Fa. Licor, Nebraska, NE, USA). El área proyectada por el dosel se determinó a partir de fotografías de cada planta y usando el software libre ImageJ, donde se delimitó el área proyectada por el dosel y se expresó en $\mathrm{cm}^{2}$. A partir de estas variables se calcularon los siguientes índices de crecimiento: área foliar específica (AFE), tasa de crecimiento del cultivo (TCC) y tasa de asimilación neta (TAN), usando las ecuaciones 1, 2 y 3 , respectivamente.

$$
\mathrm{AFE}=\mathrm{L}_{\mathrm{a}} / \mathrm{W}=\left(\left(\mathrm{L}_{\mathrm{a} 2} / \mathrm{W}_{2}\right)+\left(\mathrm{L}_{\mathrm{a} 1} / \mathrm{W}_{1}\right)\right) / 2
$$

Donde $L_{a}$ es el área foliar y $W$ el peso seco total.

$$
\mathrm{TCC}=\left(1 / \mathrm{G}_{\mathrm{a}}\right) * \mathrm{dW} / \mathrm{dT}=\left(1 / \mathrm{G}_{\mathrm{a}}\right)^{*}\left(\left(\mathrm{~W}_{2}-\mathrm{W}_{1}\right) /\left(\mathrm{T}_{2}-\mathrm{T}_{1}\right)\right)(2)
$$

Donde $G_{a}$ es el áerea proyectada por el dosel, d es la derivada, $\mathrm{W}$ el peso seco total y $\mathrm{T}$ el tiempo. 
$\mathrm{TAN}=\left(1 \mathrm{~L}_{\mathrm{a}}\right)^{*} \mathrm{dW} / \mathrm{dT}=\left(\left(\mathrm{W}_{2}-\mathrm{W}_{1}\right) /\left(\mathrm{T}_{2}-\mathrm{T}_{1}\right)\right) *\left(\left(\mathrm{LN} \mathrm{L}_{\mathrm{a} 2}-\mathrm{LN} \mathrm{L}_{\mathrm{a} 1}\right) /\left(\mathrm{L}_{\mathrm{a} 2}-\mathrm{L}_{\mathrm{a} 1}\right)\right)$

Donde $\mathrm{L}_{\mathrm{a}}$ es área foliar, d es la derivada, $\mathrm{W}$ el peso seco total, T el tiempo y LN logaritmo natural.

Además de lo anterior, se determinó cada etapa fenológica (desarrollo) del cultivo con base en la escala BBCH tomando en cuenta el número de hojas, número de ramas laterales y aparición de órgano floral. Finalmente se observó la presencia o ausencia de nódulos en raíces.

\section{Cuantificación de clorofilas}

Se determinó realizando mediciones en tres hojas completamente expandidas (sin tocar la nervadura principal) con un medidor de clorofila portátil SPAD502 (Fa. Spectrum Technologies, Aurora, IL, USA) para cada planta, al momento de cada muestreo, en plantas elegidas aleatoriamente. Este equipo determina la cantidad relativa de clorofila presente en la medición de la absorbancia de la hoja.

\section{Diseño experimental y análisis estadístico}

Se realizó un diseño en bloques completamente al azar, con cuatro bloques, cuatro camas por bloque y 18 plantas por cama, donde la unidad experimental correspondió a una planta de Phaseolus vulgaris var. Cerinza. Se establecieron cuatro tratamientos, que corresponden a tres niveles de fertilización (Químico con Triple 15, orgánico con lombricompost y compost); adicionalmente se realizó un tratamiento testigo (sin ningún tipo de fertilización): T1: testigo, T2: compost, T3: lombricompost, T4: Triple 15. E1 análisis estadístico se realizó con el programa SAS V. 9.3 (Statistical Analysis System) usando un nivel de significancia de $P \leq 0,05$. Se realizó la verificación de supuestos con las pruebas de Shapiro-Wilk (normalidad) y Bartlett (Homocedasticidad). Posteriormente se realizaron análisis de varianza ANOVA y pruebas de comparación de medias de Tukey.

\section{RESULTADOS Y DISCUSIÓN}

El estadio fenológico en que se encontraban las plantas en el momento de cada muestreo para los diferentes tratamientos y las variables que fueron significativamente diferentes mediante las pruebas de comparación de medias de Tukey, se describen en la tabla 1.

En la variable de altura de la planta (figura 1A), no se observaron diferencias significativas al inicio del ciclo sino hasta los $49 \mathrm{dds}$, donde se encontraron diferencias significativas entre el tratamiento con lombricompost respecto al compost y Triple 15 , siendo el lombricompost el que presentó los mayores valores, pero no hubo diferencias de estos respecto al testigo; a los $56 \mathrm{dds}$, los tratamientos con compost y lombricompost no presentaron diferencias significativas entre ellos, pero sí respecto al testigo y al Triple 15, siendo mayores los tratamientos orgánicos. Lo anterior es posible que se deba a la capacidad de los fertilizantes orgánicos de aumentar la disponibilidad de agua y nutrientes, lo cual permite a la planta mantener mayor turgencia y aumentar su altura, este puede ser producido por el aumento de la disponibilidad de fósforo debido a los ácidos orgánicos de los fertilizantes compost y lombricompost, tal y como señalan Arriojas (1986) y Arun-Prasad et al. (1990) quienes encontraron que la alta disponibilidad de fósforo incide en la altura de plantas leguminosas. Estos resultados también fueron obtenidos por Gómez-Álvarez et al. (2008) quienes realizaron un ensayo con plantas de $P$. vulgaris con aplicaciones de $5 \mathrm{t} \mathrm{ha}^{-1}$ de compost con contenidos de $1,2 \%$ de nitrógeno.

Se registraron diferencias significativas para peso fresco total (Fig. 1B) únicamente en el tratamiento con Triple 15 respecto del testigo al inicio del ciclo del

\begin{tabular}{|c|c|c|c|c|c|}
\hline \multirow{2}{*}{ Días despues de la siembra (dds) } & \multicolumn{4}{|c|}{ Estadio } & \multirow{2}{*}{ Variables directas significativas } \\
\hline & $\mathrm{T}_{1}$ & $\mathrm{~T}_{2}$ & $\mathrm{~T}_{3}$ & $\mathrm{~T}_{4}$ & \\
\hline 31 & 14 & 15 & 15 & 16 & Área foliar, peso seco y fresco \\
\hline 41 & 22 & 24 & 24 & 23 & No significancias \\
\hline 48 & 51 & 55 & 51 & 55 & Altura de la planta y número de botones florales \\
\hline 56 & 55 & 55 & 55 & 55 & Altura, peso fresco y seco \\
\hline
\end{tabular}



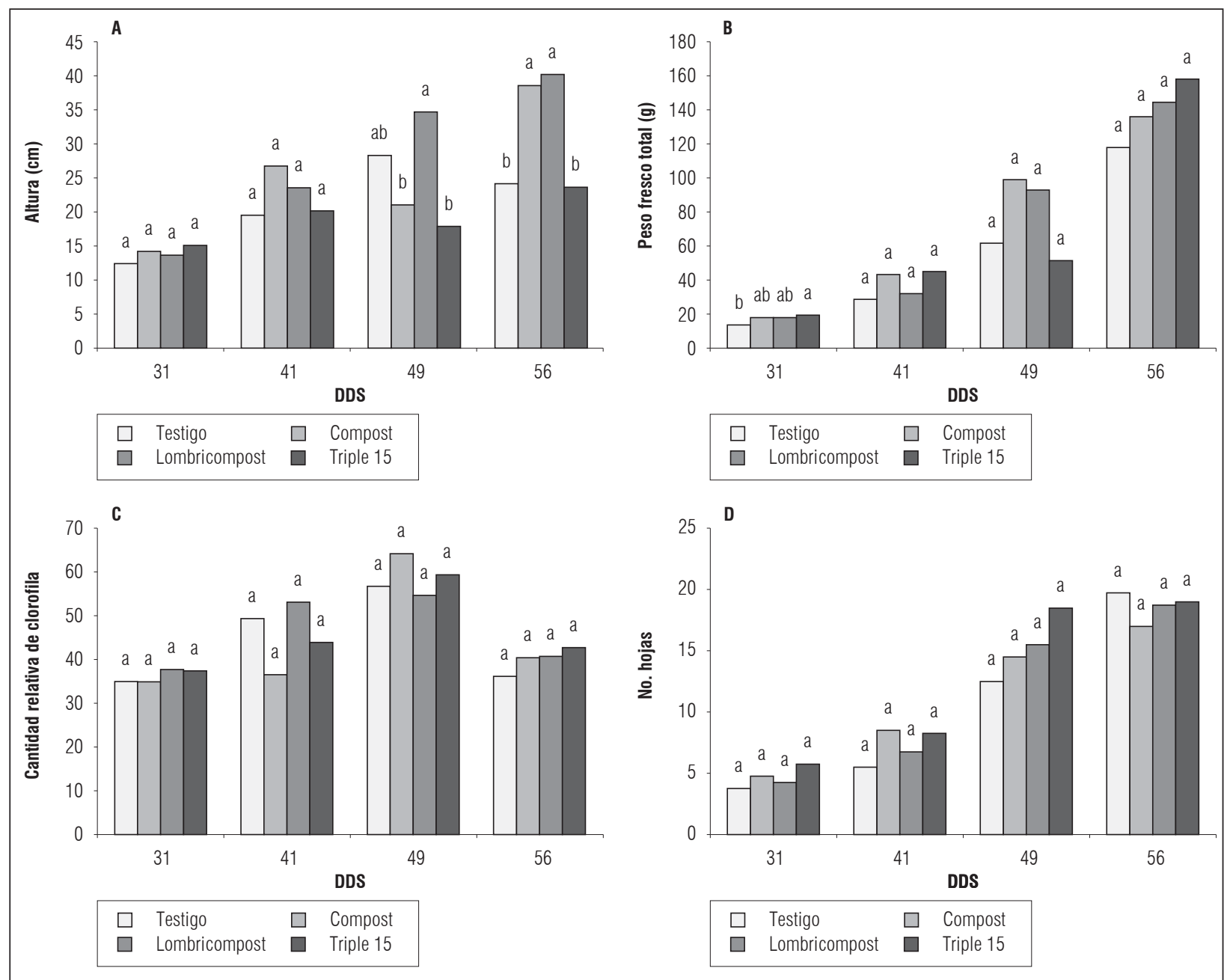

Figura 1. Efecto de tres tipos de fertilización en: (A) Altura; (B) Peso fresco; (C) Cantidad relativa de clorofila y (D) Número de hojas en Phaseolus vulgaris var. Cerinza. Promedios con letras distintas indican diferencia significativa según la prueba de comparación múltiple de Tukey $(P \leq 0,05)$.

cultivo (31 dds). Los autores sugieren que esta tendencia se debe a la alta solubilidad de los gránulos que componen el fertilizante Triple 15, lo que permitió que los nutrientes fueran disponibles más rápidamente, en especial el potasio, el cual actúa como osmorregulador permitiendo la entrada de agua a las células, lo que aumentó el peso fresco de la planta. Escobar et al. (2013) evaluaron diferentes formas de fertilización en frijol y maíz, observando un comportamiento similar al mencionado anteriormente, en donde los estadios tempranos de las plantas de frijol tratadas con fertilización química presentaron los valores más altos de peso fresco $(54.77 \mathrm{~g})$, también observaron que los valores más altos de peso fresco en la etapa reproductiva del cultivo correspondieron a las plantas tratados con fertilización orgánica.
El contenido relativo de clorofila, no presentó diferencias significativas durante el ensayo, sin embargo, se presenta una disminución para todos los tratamientos, hacia el final del ciclo experimental, posiblemente como consecuencia de la expansión del área foliar lo cual redujo la concentración de clorofila por unidad de área (Fig. 3C); contrario a lo reportado por Bedada et al. (2014), quienes observaron un aumento del contenido de clorofila en los tratamientos con aplicación de compost, debido a que la materia orgánica favorece la actividad microbiana de algunos organismos fijadores de nitrógeno en el suelo (Rhizobium), lo que genera mayor disponibilidad de nitrógeno, resultando en un mayor contenido de clorofilas. Roy et al. (2010) señalan que una aplicación de $8 \%$ de vermicompost en el sustrato incrementó el contenido de clorofila en 

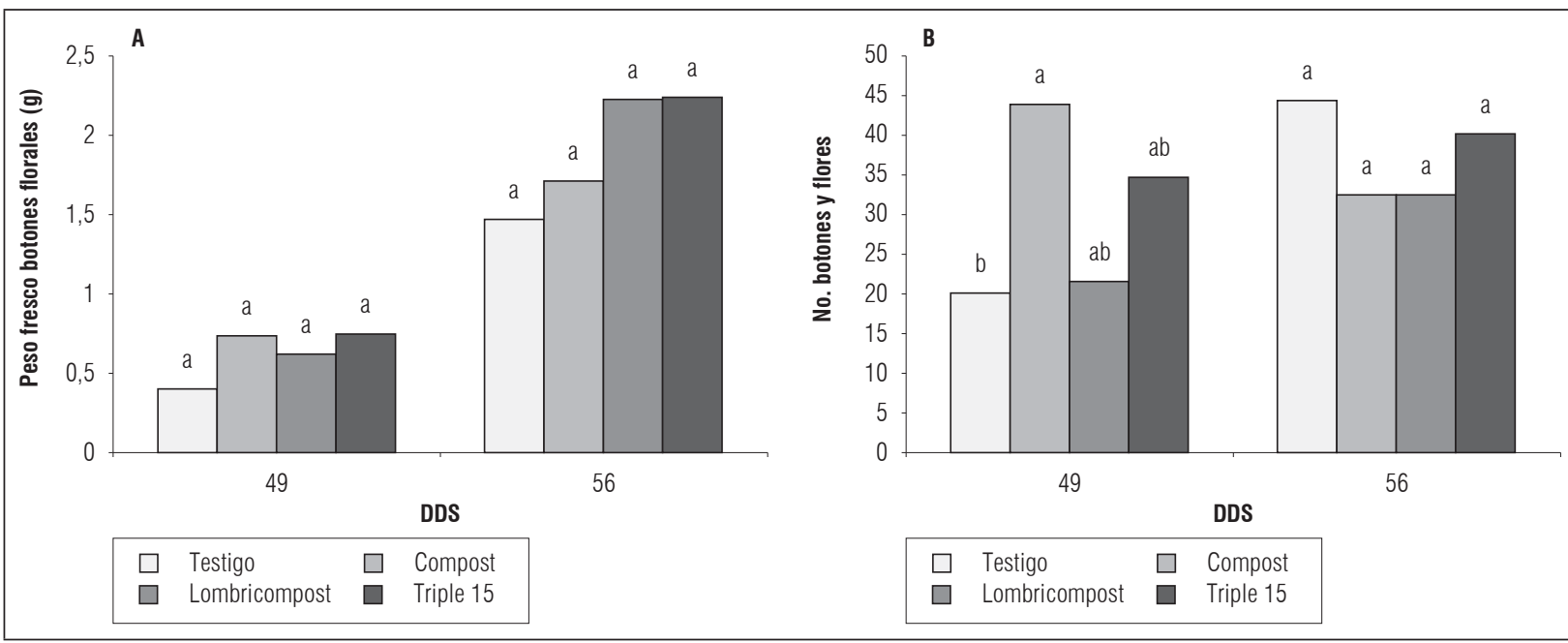

Figura 2. Efecto de la aplicación de tres tipos de fertilización en: (A) Peso fresco de botones florales y (B) Número de botones florales de Phaseolus vulgaris var. Cerinza. Promedios con letras distintas indican diferencia significativa según la prueba de comparación múltiple de Tukey $(P \leq 0,05)$.

las hojas de frijol, estando relacionado con la acumulación de biomasa.

Durante el ciclo, se llegó a etapas tempranas de la reproducción de la planta, no obstante, no se alcanzó el periodo de cosecha (Tab. 1). En ninguno de los tratamientos se presentaron diferencias significativas en el número de hojas (Fig. 1D) y el desarrollo de la planta fue normal.

La máxima etapa fenológica que se alcanzó fue 55, etapa reproductiva de generación de botones florales, los cuales surgieron a los $49 \mathrm{dds}$. En el número de botones se observaron diferencias significativas a los 49 dds, siendo las plantas testigo las que presentaron el menor valor de esta variable (Fig. 2B). Sin embargo, es posible que las diferencias sean más evidentes en el momento de realizar la cosecha, en donde se ha reportado que altas proporciones de compost y lombricompost mejoran el rendimiento de plantas de P. vulgaris, debido a que se mejoran propiedades del suelo tanto químicas (conductividad eléctrica, contenido de carbono orgánico) como físicas (humedad, temperatura) favoreciendo la toma de nutrientes por parte de la planta (Jácome et al., 2013). Respecto al peso fresco de los botones no se presentaron diferencias significativas (Fig. 2A).

En la figura 3A se observa un crecimiento progresivo del área foliar a través del tiempo, presentándose diferencias significativas en el primer muestreo entre el tratamiento con Triple 15 respecto al testigo, siendo el Triple 15 mayor en un 55,2\%. Este comportamiento puede ser resultado de la alta solubilidad del Triple 15 en el suelo, lo que generó una asimilación y absorción adecuada por parte de la planta en la evaluación inicial (Palacios, 2013). Los valores reportados para área foliar en todos los tratamientos fueron mayores a los encontrados por Maldonado y Corchuelo (1993) para P. vulgaris 'Cerinza', en donde el área foliar total fue de $172,52 \mathrm{~cm}^{2}$ a los $29 \mathrm{dds}, 449,77 \mathrm{~cm}^{2}$ a los $43 \mathrm{dds}$, $706,81 \mathrm{~cm}^{2}$ a los 50 dds y a los $57 \mathrm{dds} 1.153,45 \mathrm{~cm}^{2}$

En el área proyectada por el dosel (Fig. 3B), se observa también un incremento en los valores de esta variable a lo largo del ciclo del cultivo, no obstante, no se presentan diferencias significativas entre los tratamientos para ningún punto de muestreo, mientras que Mendoza et al. (2014) encontraron respuestas diferenciadas entre la aplicación de compost y lombricompost en el crecimiento de hojas.

En cuanto a la TCC, índice que se refiere al incremento de biomasa por unidad de tiempo (Gil et al., 2003), se observan valores altos para los tratamientos de Triple 15 y Compost, al inicio del ciclo presentándose una disminución hacia el final y siendo asintótico entre el testigo y el tratamiento con aplicación de lombricompost, sin embargo no se presentaron diferencias significativas para esta variable entre los tratamientos a lo largo del ensayo (Fig. 3C). Escobar et al. (2013) en un ensayo con Phaseolus vulgaris, evaluando tipos de fertilización, encontraron valores de TCC mayores para el tratamiento con aplicación de Triple 15 respecto al testigo. 


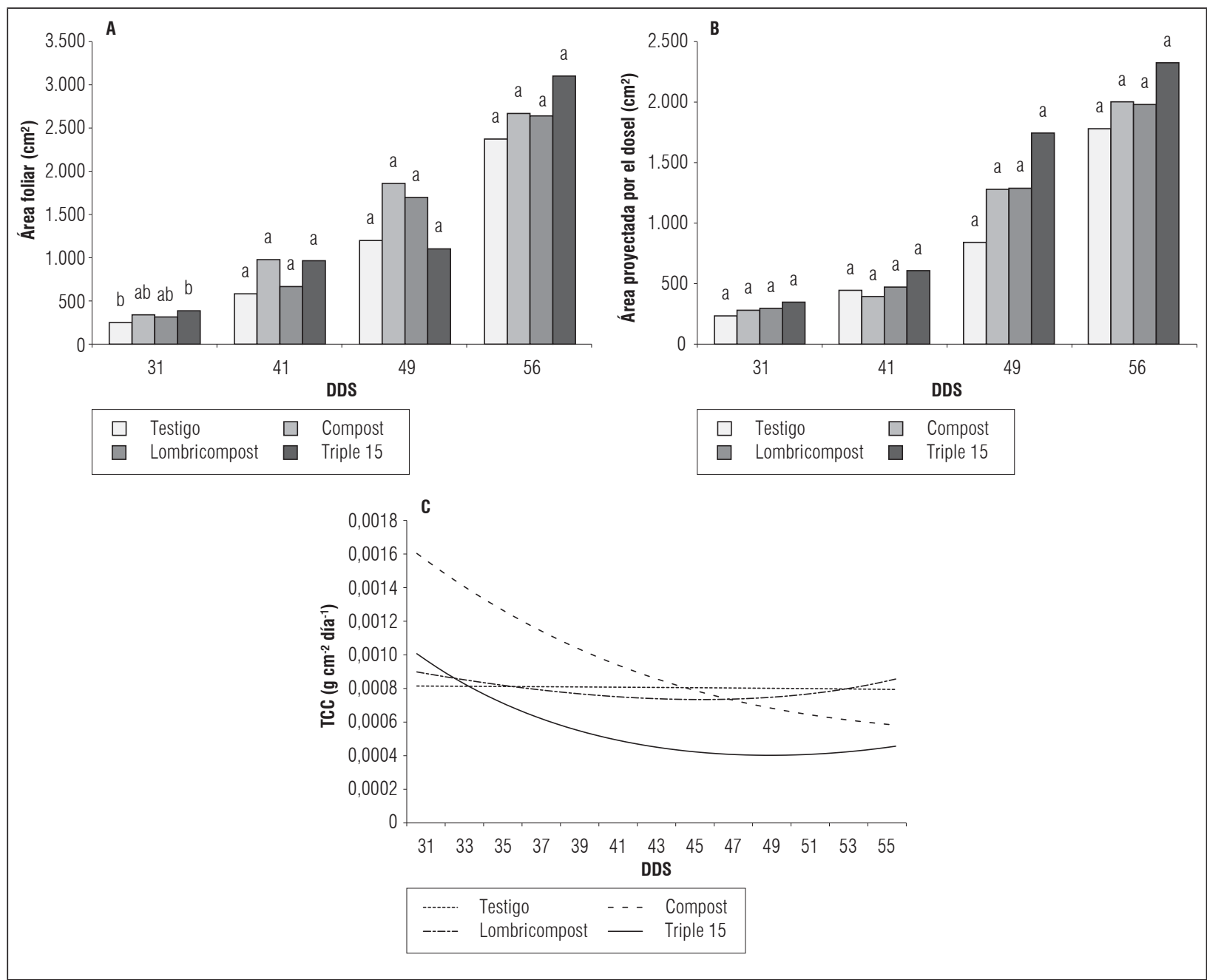

Figura 3. Efecto de tres tipos de fertilización en: (A) Área foliar, (B) Área bajo el dosel y (C) TCC de Phaseolus vulgaris var. Cerinza. Promedios con letras distintas indican diferencia significativa según la prueba de comparación múltiple de Tukey $(P \leq 0,05)$.

No se observaron diferencias significativas en la materia seca total de la planta (Fig. 4A) durante todo el ciclo de cultivo. Se alcanzó una materia seca total de 17,5 g promedio en todos los tratamientos evaluados a los 53 dds. En un estudio realizado por Maldonado y Corchuelo (1993) para Phaseolus vulgaris cv. Cerinza, mostraron que la materia seca de los tallos en función del tiempo siguió una tendencia de tipo cuadrática, mientras que para la materia seca de hojas, la tendencia fue lineal; igualmente encontraron que esta variedad, en cuanto a materia seca en tejidos foliares y caulinares, invierte una mayor cantidad de materia seca en la producción de tallos, $38 \%$ del peso total, debido a que realiza una acumulación transitoria de fotoasimilados en los tallos que son destinados a las vainas en formación, esta observación la realizaron a los 78 dds, por lo que en las plantas evaluadas en el presente estudio es posible que a los 56 dds, la acumulación de asimilados en los tallos aún se estaba llevando a cabo para ser usado al llegar a la etapa de llenado de vainas.

Respecto a la TAN, se observa un valor máximo de asimilación al inicio del cultivo, para todos los tratamientos, la cual desciende paulatinamente hasta los 56 dds. Maldonado y Corchuelo (1993) reportaron que, para esta variedad de frijol, la TAN presenta valores bajos en la primera y última etapa del cultivo y los valores más altos hacia la mitad de la fase vegetativa, lo que concuerda con el comportamiento encontrado en todos los tratamientos, ya que el primer punto de muestreo inició aproximadamente hacia la mitad del 


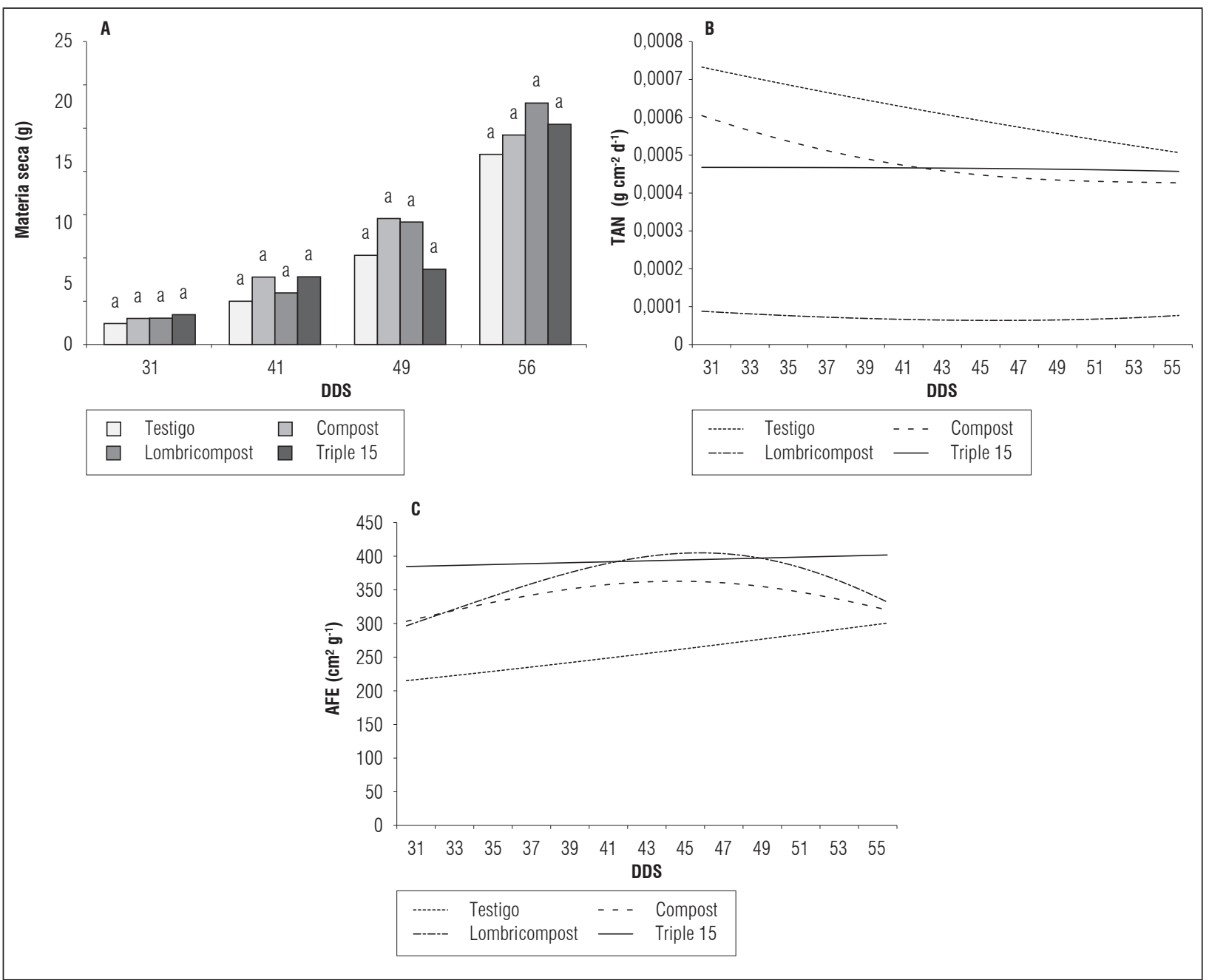

Figura 4. Efecto de tres tipos de fertilización en: (A) Materia seca total, (B) TAN y (C) AFE de Phaseolus vulgaris var. Cerinza. Promedios con letras distintas indican diferencia significativa según la prueba de comparación múltiple de Tukey $(P \leq 0,05)$.

cultivo, observándose los valores más altos y descendiendo hasta los valores más bajos a los 56 dds (Fig. 4B). Lo anterior se explica porque al inicio del ciclo de cultivo las plantas se encontraban en las primeras etapas de su desarrollo de hojas, donde la mayoría de las hojas están expuestas y por lo tanto la asimilación es máxima en este punto, al final del cultivo disminuye marcadamente la TAN por el amplio solapamiento de las hojas en el frijol, lo que dificulta la asimilación neta del $\mathrm{CO}_{2}$, proceso que según Pessanha (1980) empieza a ser determinante partir de los 20 dds.

Los valores mínimos de la TAN se reportaron para el tratamiento con lombricompost $\left(0,0002 \mathrm{~g} \mathrm{~cm}^{-2} \mathrm{~d}^{-1}\right)$ y el máximo se determinó para el tratamiento testigo $\left(0,0005 \mathrm{~g} \mathrm{~cm}^{-2} \mathrm{~d}^{-1}\right)$ a los $56 \mathrm{dds}$, sin embargo, no se encontraron diferencias significativas entre tratamientos. El suelo presentaba una alta capacidad de intercambio catiónico con $25 \mathrm{cmolc} \mathrm{kg}^{-1}$, lo que pudo haber permitido el adecuado crecimiento de las plantas incluso en ausencia de fertilización. Resultados que se soportan con lo obtenido en variables como peso fresco y área foliar, donde tampoco se observaron diferencias significativas entre tratamientos al final del ciclo experimental. Otro factor que pudo haber influido en el desarrollo de las plantas en el tratamiento testigo, es la nodulación que se observó para este tratamiento. Estudios previos reportan la disminución de nodulación en cultivos fertilizados con P y N (Onguene y Onana, 2012). Adicionalmente el análisis del suelo reveló que el contenido de carbono orgánico era de 8,4\% al inicio del experimento, lo que se 


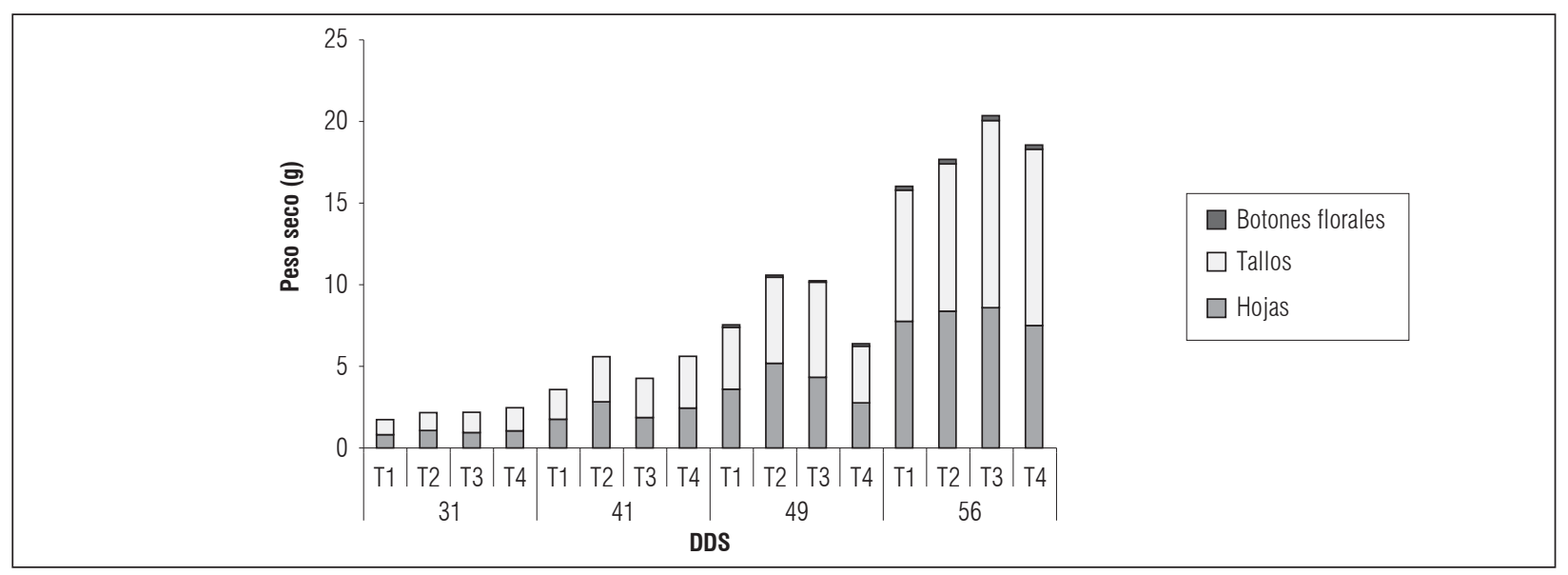

Figura 5. Distribución de materia seca en cuatro puntos de muestreo de $P$. vulgaris.

encuentra categorizado como un nivel alto para esta variable de acuerdo al suelo y al clima (ICA, 1992). Esto pudo haber favorecido el crecimiento de las plantas del tratamiento testigo, evitando que se presentaran deficiencias y en consecuencia expresando un comportamiento similar a los otros tratamientos. Lo que indica que los fertilizantes orgánicos presentaron un comportamiento similar al tratamiento de Triple 15 y testigo.

El AFE, definida como la razón entre el área foliar y el peso seco total de la hoja, es decir una medida de foliosidad de la planta con base en la materia seca de la misma (Pérez et al., 2004), no presentó diferencias significativas entre tratamientos. Generalmente el AFE presenta un comportamiento creciente al inicio del ciclo y posteriormente decrece como consecuencia de la senescencia de la planta, aunque esto varía con la intensidad lumínica, época del año o variedad que se esté trabajando. Lo reportado para la variedad de frijol Cerinza, la cual es una planta de hábito de crecimiento determinado, indica un aumento del área foliar específica al inicio y al final del ciclo, siendo este último ocasionado por la reducción en el peso de las hojas, debido a translocación de fotoasimilados y la posible remoción de elementos constituyentes del tejido foliar hacia estructuras reproductivas en formación, y una disminución del AFE a la mitad del ciclo entre los 43 y 57 dds (Maldonado y Corchuelo, 1993). No obstante, en la figura 4C, se puede observar un comportamiento en todos los tratamientos, donde hay un aumento al inicio y luego muestra un comportamiento asintótico, atribuido a que, para el final del ciclo experimental, aún no se había terminado el ciclo productivo.
Se observó que no se presentaron diferencias en la distribución de materia seca entre tratamientos de fertilización a lo largo del ciclo experimental, no obstante, se presenta una tendencia entre el tratamiento de Compost y el de Lombricompost a ser los valores más altos de materia seca total a los 49 y 56 dds, en donde se empieza a observar la aparición de botones florales (Fig. 5). La distribución presenta a partir del día 56 un aumento en el peso de las hojas con respecto al peso total de la planta, proceso con el que se prepara la planta para producir asimilados destinados a la fase reproductiva (Maldonado y Corchuelo. 1993).

\section{CONCLUSIONES}

Este estudio no determinó diferencias entre los fertilizantes orgánicos compost y lombricompost con respecto a la aplicación de Triple 15. Es necesario indagar más sobre el efecto de los abonos orgánicos en contraste con los fertilizantes químicos, para construir un esquema de ventajas y desventajas de cada uno de estos compuestos de diferente naturaleza, así como proponer puntos de equilibrio que permitan una óptima producción e involucren las virtudes de los distintos tipos de fertilizantes.

\section{REFERENCIAS BIBLIOGRÁFICAS}

Alemán, V. 2006. Efecto de niveles de Composta y hongo micorrízico arbuscular en el desarrollo y crecimiento de frijol Phaseolus vulgaris L. Tesis de maestría. Facultad de Ciencias Biológicas y Agropecuarias, Universidad de Colima. Colima, México. 
Arias, J., M. Jaramillo y T. Rengifo. 2007. Manual técnico: buenas prácticas agrícolas, en la producción de fríjol voluble. FAO, Corpoica, Gobernación de Antioquia, MANA, Centro de Investigación La Selva. Medellín, Colombia.

Arriojas, L. 1986. Leucaena leucocephala como planta forrajera. Rev. Alcance 31,169-192.

Arun-Prasad, N., T. Kapoor, P. Khatin, J. Chouham y A. Bhowmick. 1990. Effect of salts on the soil reaction and growth and dry matter yield of Leucaena leucocephala and Acacia auriculiformis in pot cultures. Indian For. 116(3), 227-232.

Bedada, W., E. Karltun, M. Lemenih y M. Tolera. 2014. Longterm addition of Compost and NP fertilizer increases crop yield and improves soil quality in experiments on smallholder farms. Agr. Eco. Env. 195, 193-201. Doi: 10-1016/j.agee.2014.06.017

Bell, F.G. 1993. Engineering treatment of soils: Soil stabilization. E \& FN Spon, London, UK. Doi: 10.4324/9780203474181

Citak, S. y S. Sonmez. 2010. Effects of conventional and organic fertilization on spinach (Spinacea oleracea L.) growth, yield, vitamin $\mathrm{C}$ and nitrate concentration during two successive seasons. Sci. Hortic. 126(4), 415-420. Doi: 10.1016/j.scienta.2010.08.010

Coral, D. y C. Molina. 2010. Manejo eficiente de nutrientes en el cultivo de frijol. Fenalce, Bogotá, Colombia.

Díaz Z., M. y M.V. Fernández. 1999. Patrones de nodulación de soja en relación con propiedades de suelo bajo tres sistemas de labranza. Rev. Fac. Agron. 104(1), 53-60.

Drinkwater, L.E., D.K. Letourneau, F. Workneh, A.H.C. van Bruggen y C. Shennan. 1995. Fundamental differences between conventional and organic tomato agroecosystems in California. Ecol. Appl. 5, 1098-1112. Doi. 10.2307/2269357

Edwards, C.A. y J.R. Lofty. 1982. Nitrogenous fertilizer and earthworm population in agricultural soils. Soil Biol. Biochem. 14: 515-521. Doi: 10.1016/0038-0717(82)90112-2

Escobar, N., J. Mora y N. Romero. 2013. Respuesta agronómica de Zea mays L. y Phaseolus vulgaris L. a la fertilización con Compost. Rev. Lun. Az. 37:18-29.

Fenalce (Fondo Nacional Cerealista). 2004. Sensibilidades del sector cerealista y de leguminosas: Frijol. En: http://fenalce.org/archivos/frijoldmlm.pdf; consulta: abril de 2017.

Gentile, R., B. Vanlauwe, A. Kavoo, P. Chivenge y J. Six. 2010. Residue quality and $\mathrm{N}$ fertilizer do not influence aggregate stabilization of $\mathrm{C}$ and $\mathrm{N}$ in two tropical soiIs with constrasting texture. Nutr. Cycl. Agroecosyst. 88(1), 121-131. Doi: 10.1007/s10705-008-9216-9
Gil, P., J. Orozco, E. Gutiérrez, A. Espinoza y S. Rodríguez. 2003. Análisis de crecimiento de variedades de algodón transgénicas y convencionales. En: http://www. uaaan.mx/DirInv/Rdos2003/cultbasicos/analisis.pdf, consulta: abril de 2017.

Gómez-Álvarez, R., G. Lázaro-Jerónimo y J. Leon-Nájera. 2008. Producción de frijol (Phaseolus vulgaris) y rábano (Rhabanus sativus) en huertos biointensivos en el trópico húmedo de tabasco. Univ. Cienc. 24(1), 11-20.

Hernández, V., M. Vargas, J. Muruaga, S. Hernández y N. Mayek, N. 2013. Origen, domesticación y diversificación del frijol común. Avances y perspectivas. Rev. Fitotec. Mex. 36(2), 95-104.

Hernández, T., C. Chocoano, J. Moreno y C. García. 2014. Towards a more sustainable fertilization: Combined use of Compost and inorganic fertilization for tomato cultivation. Agric. Ecosyst. Environ. 196, 178-184. Doi: 10.1016/j.agee.2014.07.006

Hoitink, H.A.J. y M.J. Boehm. 1999. Biocontrol within the context of soil microbial communities: a substrate-dependent phenomenon. Annu. Rev. Phytopathol. 37, 472-446 Doi: 10.1146/annurev.phyto.37.1.427

ICA. 1992. Fertilización en diversos cultivos. Quinta aproximación. Manual de asistencia técnica No 25. Instituto Colombiano Agropecuario, Bogotá, Colombia.

Jácome, A., W. Peñarete y M. Daza. 2013. Fertilización orgánica e inorgánica de fríjol (Phaseolus vulgaris L.) en suelo inceptisol con propiedades ándicas. Ing. Recur. Nat. Ambient. 12, 59-67.

Jedidi, N., A. Hassen, O. Van Cleemput y A. M'Hiri. 2004. Microbial biomass in a soil amended with different types of organic wastes. Waste Manag. Res. 22, 93-99. Doi: 10.1177/0734242X04043930

Kapkiyai, J.J., P.L. Woomer, N.K. Karanja, J.N. Qureshi y P.C. Smithson. 1999. Soil organic matter and nutrient dynamics in a Kenyan nitisol under long-term fertilizer and organic input management. Soil Biol. Biochem. 31, 1773-1782. Doi: 10.1016/S0038-0717(99)00088-7

Kwabiah, A.B., N.C. Stoskopf, C.A. Palm, R.P. Voroney, M.R. Rao y E. Gacheru. 2003. Phosphorus availability and maize response to organic and inorganic fertilizer inputs in a short term study in western Kenya. Agric. Ecosys. Environ. 95, 49-59. Doi: 10.1016/ S0167-8809(02)00167-6

Leifeld, J., S. Siebert e I. Kogel-Knabner. 2002. Changes in the chemical composition of soil organic matter after application of compost. Eur. J. Soil Sci. 53, 299-309. Doi: 10.1046/j.1351-0754.2002.00453.x

Maldonado, G y G. Corchuelo. 1993. Dinámica de crecimiento de dos variedades de frijol (Phaseolus vulgaris L.). Agron. Col. 10(2) ,114-121.

Miranda, S. 1967. Origen de Phaseolus vulgaris L. (frijol común). Agrocienc. 1(2), 99-109. 
Onguene, C. y L. Onana. 2012. Starter N and P fertilizers have dissimilar effects on native mycorrhizal and bradyrhizobial symbiosis of four promiscuous soybean varieties in acid soils of Cameroon. Tropicul. 30(3), 125-132.

Palacios, M. 2013. Evaluación de la respuesta a la fertilización química y orgánica de la uvilla Physalis peruviana L. en la provincia de Imbabura cantón Antonia Ante. Facultad de Ingeniería Química y Agroindustrial, Escuela Politécnica Nacional. Quito, Ecuador.

Palma, R.M., M. Rimolo, M.I. Saubidet y M.E. Conti. 1997. Influence of tillage system on denitrification in maize-cropped soils. Biol. Fert. Soils 25, 142-146. Doi: 10.1007/s003740050294

Pérez, J., E. García, J. Enríquez, A. Quero, J. Pérez y A. Hernández. 2004. Análisis de crecimiento, área foliar específica y concentración de nitrógeno en hojas de pasto "mulato" (Brachiaria híbrido, cv.). Téc. Pecu. Méx. 42(3) ,447-458. Doi: 10.22319/rmcpv\%251397

Pessanha. 1980. Estudio sobre mixturas de cultivares de feijao (Phaseolus vulgaris L.). Tesis de doctorado. Universidad Federal de Vicosa. Minas Gerais, Brasil.
Roy, S., K. Arunachalam, B. Kumary A. Arunachalam. 2010. Effects of organic amendments of soil on growth and productivity of three common crops viz. Zea mays, Phaseolus vulgaris and Abelmoschus esculentus. Appl. Soil Ecol. 45(2), 78-84. Doi: 10.1016/j.apsoil.2010.02.004

Santana, G. y G. Mahuku. 2002. Diversidad de razas de Colletotrichum lindemuthianum en Antioquía y evaluación de germoplasma de frijol crema-rojo por resistencia a antracnosis. Agron. Mesoamer. 13(2), 95-103. Doi: 10.15517/am.v13i2.12024

Schjonning, P. y B.T. Christensen. 1994. Physical and chemical properties of a sandy loam receiving animal manure, mineral fertilizer or no fertilizer for 90 years. Eur. J. Soil Sci. 45, 257-268. Doi: 10.1111/j.1365-2389.1994. tb00508.x

Ulloa, J., P. Rosas, J. Ramírez y B. Ulloa. 2011. El frijol (Phaseolus vulgaris): su importancia nutricional y como fuente de fitoquímicos. Rev. Fuente 3(8), 5-9.

Wander, M.M., S.J. Traina, B.R. Stinner y S.E. Peters. 1994. Organic and Conventional Management Effects on Biologically Active Soil Organic Matter Pools. Soil Sci. Soc. Am. J. 58, 1130-1139. Doi:10.2136/ sssaj1994.03615995005800040018x 